\section{Einführung in den Altersbegriff}

Ein höheres Lebensalter geht bei höherentwickelten Lebewesen mit Seneszenz einher, also mit sichtbaren oder messbaren Stigmata eines Alterungsprozesses, der natürlich, also „physiologisch“ erscheint. Das organübergreifende Merkmal des physiologischen Alterungsprozesses ist der Verlust der funktionellen Reserve der einzelnen Organe; Resnick und Marcantonio prägten den Begriff „Homoiostenose“. Ein Grundproblem bei der wissenschaftlichen Analyse derartiger „physiologischer" Alterungsprozesse besteht in der Abgrenzung zu „pathologischen“ Prozessen im Rahmen der Entstehung von Alterskrankheiten, die sich auf dem Boden vorbestehender Alterungsprozesse entwickeln. Als harter Endpunkt des biologischen Alterns kann die Mortalität („life span“) angesehen werden, während die Gesundheitsspanne („health span“) weniger gut fassbar ist. Unter den verschiedenen Definitionen des Alterungsprozesses sind daher diejenigen am besten operationalisiert, die auf den Endpunkt „Sterblichkeit“ abheben, wie die des späteren Nobel- preisträgers Peter Medawar, der 1952 vorschlug, Altern sei die Summe der Veränderungen, durch die die Wahrscheinlichkeit zu versterben zunehmend erhöht wird.

Ein Mensch von mehr als 75 Jahren gilt gemeinhin als alt, auch wenn die Alterungsprozesse zu einer erheblichen phänotypischen Diversifizierung führen. Diese Phänomenologie des Alterns wird üblicherweise unter Heranziehung von Biomarkern des Alterungsprozesses beschrieben, einen Goldstandard zur Ermittlung des „biologischen Alters“ im Vergleich zum „biografischen Alter" gibt es jedoch nicht.

Die Vision einer „Geroscience“ geht dahin, dass durch Hintanhalten physiologischer Alterungsprozesse auch das Auftreten altersbezogener Erkrankungen verzögert werden könnte.

\section{Interessenkonflikt}

Vortragshonorare von Firmen Bayer, BristolMyers Squibb/Pfizer, Novartis
Autorinnen/Autoren

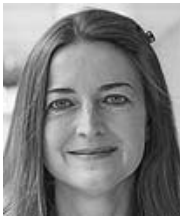

Ursula Müller-Werdan

Direktorin der Klinik für Geriatrie und Altersmedizin der Charité - Universitätsmedizin Berlin und Ärztliche Leitung/Med. Geschäftsführung EGZB

\section{Korrespondenzadresse}

Prof. Dr. Ursula Müller-Werdan

Direktorin der Klinik für Geriatrie und Altersmedizin der Charité - Universitätsmedizin Berlin

und

Ärztliche Leitung/Medizinische Geschäftsführung des EGZB

Reinickendorfer Straße 61

13347 Berlin

ursula.mueller-werdan@charite.de

\section{Literatur}

[1] Medawar PB. An Unsolved Problem of Biology. London: H. K. Lewis; 1952

[2] Müller-Werdan U. Physiologische Veränderungen im Alter: In: Anästhesie beim geriatrischen Patienten. Zink W, Bernhard G, Zausig Y, Heppner H], Hrsg. Berlin: De Gruyter: 2019 [im Druck]

[3] Resnick NM, Marcantonio ER. How should clinical care of the aged differ? Lancet 1997; 350: $1157-1158$ 